\begin{tabular}{|c|l|}
\hline Title & $\begin{array}{l}\text { Electric conductance of metal nanowires at mechanically controll able break junctions under electrochemical potential } \\
\text { control }\end{array}$ \\
\hline Author(s) & Kiguchi, Manabu; Sekiguchi, Nobuo; Murakoshi, Kei \\
\hline Citation & $\begin{array}{l}\text { Surface Science, 601(22), 5262-5265 } \\
\text { https://doi.org/10.1016/.Susc.2007.04.218 }\end{array}$ \\
\hline Issue Date & 2007-11-15 \\
\hline Doc URL & http://hdl.handle.net/2115/32316 \\
\hline Type & article (author version) \\
\hline File Information & p51-kiguti-v3.pdf \\
\hline
\end{tabular}

Instructions for use 


\section{Electric conductance of metal nanowires at mechanically controllable break junctions under electrochemical potential control}

Manabu Kiguchi ${ }^{1}$, Nobuo Sekiguchi, Kei Murakoshi

Division of Chemistry, Graduate School of Science, Hokkaido University, Kita 10, Nishi 8, Sapporo, Hokkaido, 060-0810, Japan

\section{Abstract}

We have developed the mechanically controllable break junction setup with an electrochemical cell (EC-MCBJ) to measure the electric conductance of metal nanowires under electrochemical potential control. The electric conductance of $\mathrm{Au}$ nanowires was investigated in $0.1 \mathrm{M} \mathrm{Na}_{2} \mathrm{SO}_{4}$ solution using EC-MCBJ. The conductance of the Au nanowires was quantized in units of $G_{0}$ $\left(=2 e^{2} / h\right)$, showing clear features in the conductance histogram. The atomic contact with a specific conductance value was kept for more than $5 \mathrm{sec}$, indicating the relatively high stability of the present EC-MCBJ system.

Keywords: Electrical transport, Electrochemical methods, Nanowire, Gold

\footnotetext{
${ }^{1}$ Correspondence author: Manabu Kiguchi; Address: Division of Chemistry, Graduate School of Science, Hokkaido University, Kita 10, Nishi 8, Sapporo, Hokkaido, 060-0810, Japan; TEL\&FAX+81 11706 4811; E-mail: kiguti@sci.hokudai.ac.jp
} 


\section{Introduction}

As materials and devices shrink down to the atomic scale, many interesting quantum phenomena begin to emerge. One example is conductance quantization in nanowires. Electrical conductance through a metal nanowire on an atomic scale is expressed by $G=2 e^{2} / h \sum T_{i}$ where $T_{i}$ is the transmission probability of the $i$-th conductance channel, $e$ is the electron charge, and $h$ is Planck's constant [1]. $G_{o}=2 e^{2} / h$ is the unit of the quantized conductance. The conductance quantization behavior of metals has been studied using a scanning tunneling microscope (STM), conducting-tip atomic force microscopes (cAFM), and mechanically controllable break junctions (MCBJ) [1].

The MCBJ technique was first proposed by Moreland and Ekin for the fabrication of tunable tunnel junctions [2]. Later, the method was refined to be applied toward the investigation of stable single-atom contacts [3]. The principle of a MCBJ is shown in Fig. 1. Two wires are mounted on a flexible substrate to be close to each other. By bending of the substrate with a piezo element, the distance between two electrodes can be controlled precisely. Since both wires are fixed to the same substrate, the junction is insensitive to external vibrations. Therefore, the junction has high stability, which enables various fine measurements, such as shot noise measurements and point contact spectroscopy [4-7]. Because of these advantages, experiments with MCBJ have been performed in ultra high vacuum (UHV), in air, under inert gas, atmosphere, in solution, etc [1,6-8].

Recently, preparation of metal nanowires under electrochemical potential control has attracted wide attention [9-13]. Electrochemical potential determines the potential energy of electrons in metal nanowires, resulting in control of the bonding strength between the metal atoms in the wires and the interaction between the metals and molecules in the surrounding medium. These characteristics lead to successful fabrication of very stable metal nanowires, which cannot be prepared in UHV. Relatively stable $\mathrm{Au}[9], \mathrm{Ag}, \mathrm{Cu}, \mathrm{Pb}[10], \mathrm{Fe}, \mathrm{Co}, \mathrm{Ni}[11]$, and $\mathrm{Pd}[12]$ metal nanowires were fabricated in solution at room temperature with the aid of electrochemical potential control. Furthermore, the stability and structure of Au mono atomic wires was controlled by the electrochemical potential [13]. 
Despite the advantage of the MCBJ, there have been few studies on metal nanowires under electrochemical potential control using MCBJ. In the conventional MCBJ setup, a large area of the metal wire is exposed to an electrolyte solution. In this case, the Faradic current between the two electrodes interferes with the conductance measurement of metal nanowires under electrochemical potential control. The Faradic current can be easily reduced by coating the tip with wax in the case of the electrochemical STM (EC-STM) setup. Therefore, most of the previous studies have been done with EC-STM. However, the stability of the nanowire fabricated with EC-STM is relatively low. It is difficult to keep the nanowire stable on a time scale which permits the systematic variation of parameters such as electrochemical potential or magnetic field. Thus, in the present study we have developed the MCBJ setup with an electrochemical cell to measure the electric conductance of metal nanowires under electrochemical potential control.

\section{Experimental Setup}

The bending beam was a plate of phosphor bronze about $1 \mathrm{~mm}$ thick, $20 \mathrm{~mm}$ long and $8 \mathrm{~mm}$ wide. The surface of the plate was insulated with a thin polymer foil (Kapton). The center of the 1 $\mathrm{mm}$ diameter Au wire was notched by a surgical knife to create a weak spot. Then, the wire was glued onto the bending beam by two drops of epoxy adhesive (Stycast 2850FT). The distance between the drops was manually reduced under the microscope. After complete polymerization of Stycast, the drops and the metal wire were covered with wax, except the notched area of the wire. The whole assembly was mounted on a three-point bending mechanism, consisting of a stacked piezo-element (AE0505D08: NEC Co.) and two fixed counter supports. By bending the substrate, the top surface of the substrate was expanded, and thus the wire was elongated. At some critical strain, the wire finally broke, forming two separated electrodes. By relaxing the bending of the substrate, the contact could be re-established. As a result of the chosen geometry, the setup itself acts as a reduction gear for the motion of the piezo element $(\delta x)$ with respect to the relative displacement of the two electrodes $(\delta y)$. For the ideal case of homogeneous strain in the bending 
beam, the displacement ratio $(r)$ between $\delta y$ and $\delta x$ is given by $r=\frac{\delta y}{\delta x}=\frac{6 t u}{l^{2}}$, where $t$, $u$ and $l$ are thickness of the bending beam, the distance between the epoxy droplets and the distance between the two counter supports, respectively $[1,6,7]$. The displacement ratio was $5 \times 10^{-3}$ for the present setup, with $l=20 \mathrm{~mm}, t=1 \mathrm{~mm}, u=0.3 \mathrm{~mm}$.

Figure 2 (a) shows a photograph of the EC-MCBJ setup with the electrochemical cell. Coarse approach of the piezo element to the substrate was accomplished by a screw mechanism, controlled by a wheel. The fork-blade mechanism was used to mechanically decouple the axle from the screw mechanism in order to reduce the mechanical vibration. Figure 2 (b) shows the photograph of the electrochemical cell made of polychlorotrifluoroethylene (Diflon). The cell was tightly pressed against the substrate via springs. The electrochemical potential of the metal wire (working electrode: W.E.) was controlled with respect to a $\mathrm{Ag} / \mathrm{AgCl}$ reference electrode (R.E.) using a four-electrode potentiostat (twin potentiostat HECS990C: Fuso Co.). A $0.50 \mathrm{~mm}$ diameter Pt wire was used as a counter electrode (C.E.). The conductance of the metal nanowire was determined by the current passing through two working electrodes under a fixed potential difference between the electrodes. Figure 3 shows the block diagram of the MCBJ setup with an electrochemical cell, which enables us to measure the electric conductance of metal nanowires under electrochemical potential control. A personal computer (PC) controlled the movement of the piezo element via a high voltage source and a standard data-acquisition board (National Instruments) installed in a PC. The electric current was recorded via the potentiostat and standard data-acquisition board.

\section{Experimental Results}

Figure 4 shows the cyclic voltammogram of $\mathrm{Au}$ electrodes in $0.1 \mathrm{M} \mathrm{Na}_{2} \mathrm{SO}_{4}$ at scan rate of 50 $\mathrm{mV} / \mathrm{s}$ measured with the EC-MCBJ setup. A reduction current due to hydrogen evolution and an oxidation current due to oxygen evolution were observed in the cyclic voltammogram. The cyclic voltammogram measured with the present setup was similar to that obtained with the conventional electrochemical cell [14], indicating that the electrochemical potential of the Au nanowire was 
precisely controlled by this MCBJ setup. The effect of the Faradic current on the conductance measurement of the metal nanowire was evaluated from the cyclic voltammogram. In the conductance measurement, the electrochemical potential of the Au electrodes was kept between -1 $\mathrm{V}$ and $0.5 \mathrm{~V}$. In this potential regime, the Faradic current was suppressed to be less than $0.3 \mu \mathrm{A}$. The electric current that flows through a $\mathrm{Au}$ mono atomic contact $\left(1 \mathrm{G}_{0}\right)$ is $1.5 \mu \mathrm{A}$ when the potential difference between two working electrodes (bias voltage) is $20 \mathrm{mV}$ (typical value of the present measurement). The electric current of $1.5 \mu \mathrm{A}$ is five times larger than the Faradic current of $0.3 \mu \mathrm{A}$. Thus, the effect of the Faradic current could be neglected in the conductance measurement of the metal nanowires using the present EC-MCBJ setup.

Conductance measurements using EC-MCBJ were carried out at the breaking process under an applied bias voltage of $20 \mathrm{mV}$ between the two working electrodes. The piezo ramp amplitude $(\delta x)$ was $2.1 \mu \mathrm{m}$, which corresponded to a relative displacement of the two electrodes $(\delta y)$ of $10 \mathrm{~nm}$, assuming that the displacement ratio $(\delta y / \delta x)$ was $5 \times 10^{-3}$. Since the piezo element was moved at $6 \mathrm{~Hz}$, the displacement rate of the electrodes was $60 \mathrm{~nm} / \mathrm{s}$. Figure 5(a) shows the conductance trace of the $\mathrm{Au}$ nanowire in $0.1 \mathrm{M} \mathrm{Na}_{2} \mathrm{SO}_{4}$ solution when the electrochemical potential of the $\mathrm{Au}$ nanowire was kept at $+0.5 \mathrm{~V}$. The conductance decreased in a stepwise fashion with each step occurring at integer multiples of $\mathrm{G}_{0}$. The conductance of the last plateau was $1 G_{0}$, which corresponded to that of a contact with a single Au atom. Conductance quantization behavior of $\mathrm{Au}$ could be successfully observed with the MCBJ setup. However, the residue current and conductance fluctuation were still large with the present EC-MCBJ setup, and an improvement in the setup is in progress in our laboratory. We found that the residue current was proportional to the electrode area exposed to the solution. Our idea is that the exposed area might be drastically reduced by covering the whole metal wire with wax. Only fracture surfaces are expected to be exposed to the solution. With regard to the conductance fluctuation, it mainly originated from the electrical noise, although genuine conductance fluctuation might be observed in the conductance trace. Since the residue current interferes with the conductance measurements, the reduction of residue current would also 
decrease the conductance fluctuation caused by electric noise.

Figure 5 (b) shows the conductance histogram of the Au nanowires in $0.1 \mathrm{M} \mathrm{Na}_{2} \mathrm{SO}_{4}$ solution at an electrochemical potential of $+0.5 \mathrm{~V}$. A conductance histogram was obtained from 5000 individual conductance traces. In the conductance histogram, well-defined peaks appear near integer multiples of $G_{0}$. The conductance histogram obtained at EC-MCBJ was similar to that with EC-STM [13], indicating that the conductance quantization behavior of metal nanowires could be successfully observed using the present EC-MCBJ setup. While the conductance quantization behavior of Au generally shows little dependence on the conditions (in air, UHV, etc), due to the low reactivity of $\mathrm{Au}[1]$, the conductance histograms changed with the electrochemical potential in solution. A detailed investigation on the electrochemical potential dependence is in progress in our laboratory.

Finally, the stability of the metal nanowires was compared between the EC- MCBJ and STM setups. Figure 6 shows the time course of the conductance of the Au point contact. The contact could be kept at a unit of the quantized conductance value $\left(1 G_{0}\right.$ in Fig. 6) for more than 5 sec by stopping the movement of the piezo element. The lifetime of $5 \mathrm{sec}$ is much longer than that of the $\mathrm{Au}$ point contact fabricated with STM. The stability of EC-MCBJ allows for various fine measurements, such as current-voltage characteristics, electrochemical potential or magnetic field dependence, which would drastically facilitate the detailed study of metal nanowires in solution.

\section{Conclusion}

We have developed a mechanically controllable break junction setup with an electrochemical cell (EC-MCBJ) to measure the electric conductance of metal nanowires under electrochemical potential control. A four-electrode potentiostat was used to control the electrochemical potential of the metal nanowires for the measurement of the conductance of the metal nanowires. Using this setup, the electric conductance of $\mathrm{Au}$ nanowires was investigated in $0.1 \mathrm{M} \mathrm{Na}_{2} \mathrm{SO}_{4}$ under electrochemical potential control. Conductance quantization behavior was observed for the Au 
nanowires. A metal nanowire with a specific conductance value persisted more than $5 \mathrm{sec}$, indicating the relatively high stability of the present EC-MCBJ system.

\section{Acknowledgements}

We would like to express our sincere gratitude to Prof. J.M. van Ruitenbeek in Leiden University and Mr. T. Kato in Hokkaido University. This work was partially supported by a Grant-in-Aid for Scientific Research A (No. 16205026) and Grant-in-Aid for Scientific Research on Priority Areas "Electron transport through a linked molecule in nano-scale" (No. 17069001) from MEXT. 


\section{References}

[1] N. Agrait, A.L. Yeyati, and J.M. van Ruitenbeek, Physics Reports 377 (2003) 81.

[2] J. Moreland and J.W. Ekin, J. Appl. Phys. 58, (1985) 3888

[3] A.I. Yanson, G.R. Bollinger, H.E. van den Brom, N. Agrait, and J.M. van Ruitenbeek, Nature 395 (1998) 783.

[4] D. Djukic and J.M. van Ruitenbeek, Nano Lett. 6 (2006) 026806.

[5] R.H.M. Smit, Y. Noat, C. Untiedt, N. D. Lang, M.C. van Hemert and J.M. van Ruitenbeek, Nature 419 (2002) 906.

[6] J.M. van Ruitenbeek and D, Djukic, in: "Single Molecule Electronics", M. Reed, editor, Oxford University Press, in press.

[7] J.M. van Ruitenbeek, E. Scheer and H. Weber, in: "Introducing Molecular Electronics", G. Cuniberti, G. Fagas, and K. Richter, eds., Springer Lecture Notes in Physics (2005) 253.

[8] L. Grüter, M.T. González, R. Huber, M. Calame, and C. Schönenberger, Small 1 (2005) 1067.

[9] C. Shu, C. Z. Li, H. X. He, A. Bogozi, J. S. Bunch, and N. J. Tao, Phys. Rev. Lett. 84, 5196 (2000).

[10] J. Li, T. Kanzaki, K. Murakoshi, Y. Nakato, Appl. Phys. Lett. 81, 123 (2002).

[11] M. Kiguchi, T. Konishi, and K. Murakoshi, Appl. Phys. Lett. 87 (2005) 043104.

[12] M. Kiguchi and K. Murakoshi, Appl. Phys. Lett. 88 (2006) 253112.

[13] M. Kiguchi, T. Konishi, and K. Murakoshi, Phys. Rev. B 73 (2006) 125406.

[14] J. Bockris and S. Khan, Surface electrochemistry, Plenum, NY (1993). 


\section{Figures captions}

Fig. 1: Schematic side view of the MCBJ setup with coating wax (1), notched Au wire (2), two fixed counter supports (3), polymer foil (4), bending beam of phosphor bronze (5), epoxy adhesive (6), and piezo element (7).

Fig.2: (a) Photograph of the EC-MCBJ setup for measuring the electrical conductance of the metal nanowire under electrochemical potential control; (b) Electrochemical cell (E.C.) with counter electrode (C.E.) and reference electrode (R.E.). The cell was pressed against the substrate via springs.

Fig. 3: A block diagram of the EC-MCBJ setup for the measurement of electrical conductance of the metal nanowire under electrochemical potential control.

Fig. 4: Cyclic voltammogram of the $\mathrm{Au}$ electrodes in $0.1 \mathrm{M} \mathrm{Na}_{2} \mathrm{SO}_{4}$ measured using the MCBJ setup.

Fig. 5: Typical conductance trace (a) and conductance histograms (b) of a Au nanowire in $0.1 \mathrm{M}$ $\mathrm{Na}_{2} \mathrm{SO}_{4}$ measured using the EC-MCBJ setup. The conductance was at $+0.5 \mathrm{~V}$.

Fig. 6: Time course of the conductance of the $\mathrm{Au}$ nanowire in $0.1 \mathrm{M} \mathrm{Na}_{2} \mathrm{SO}_{4}$ at $+0.0 \mathrm{~V}$. 
Figure1

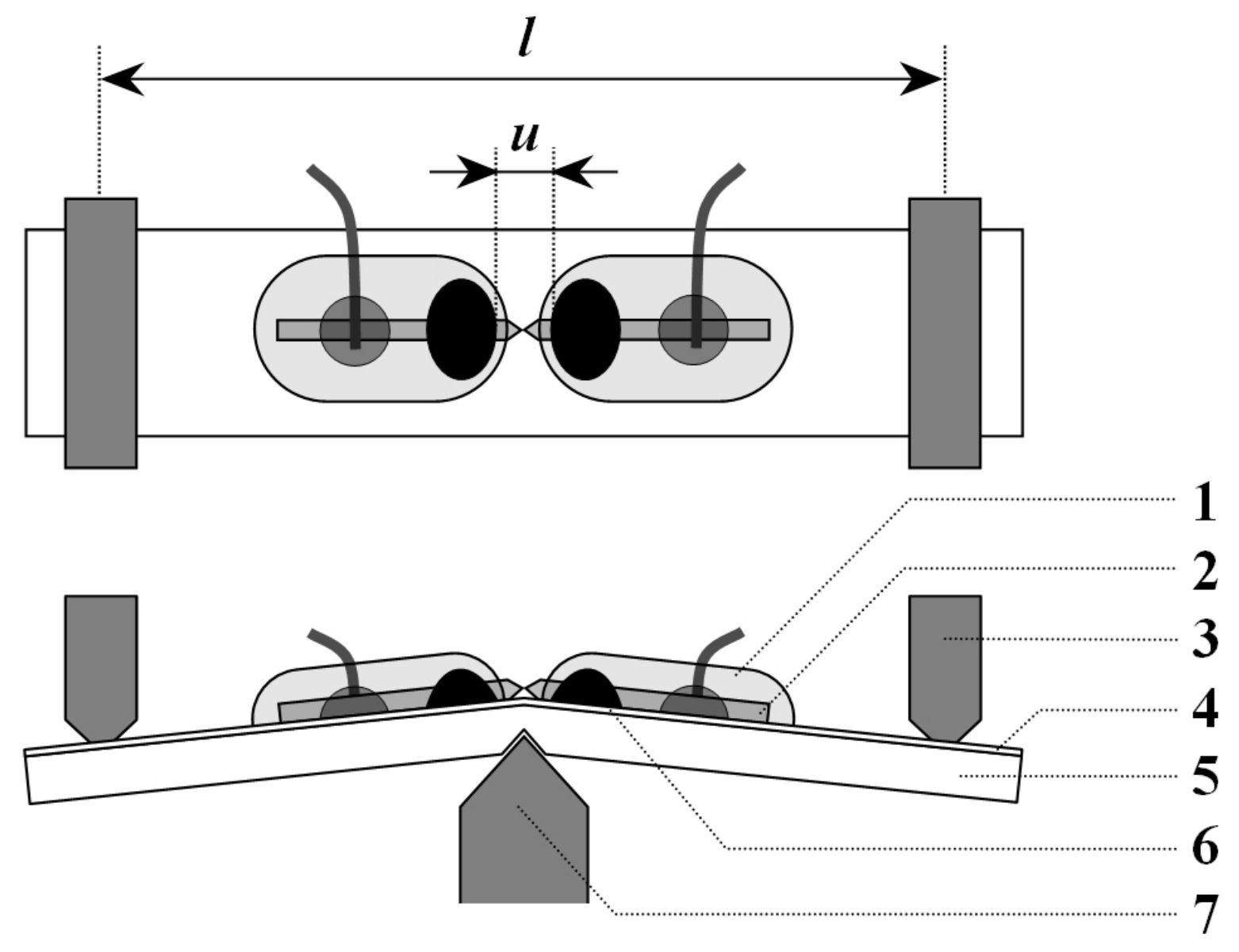


Figure2
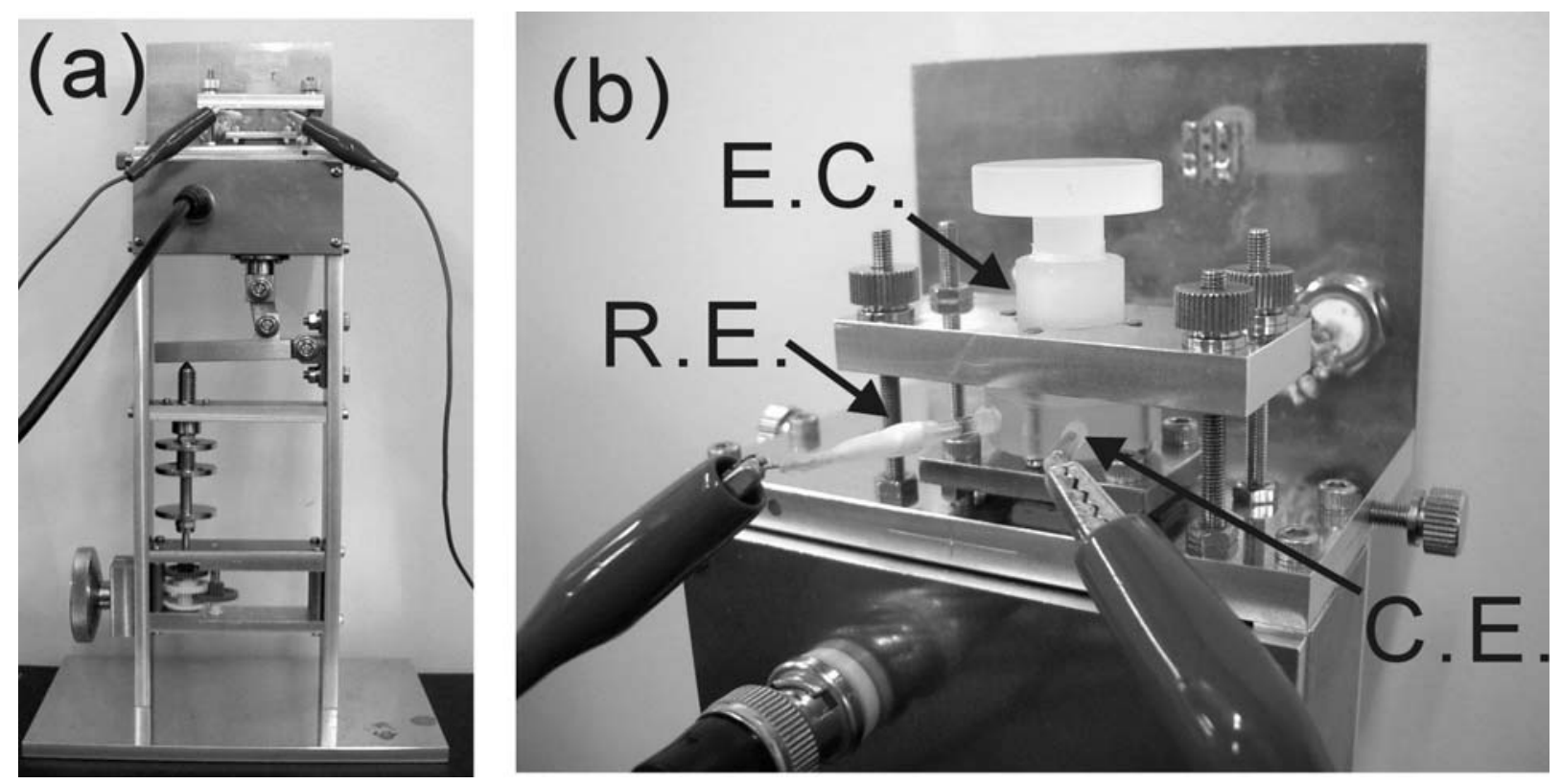
Figure3

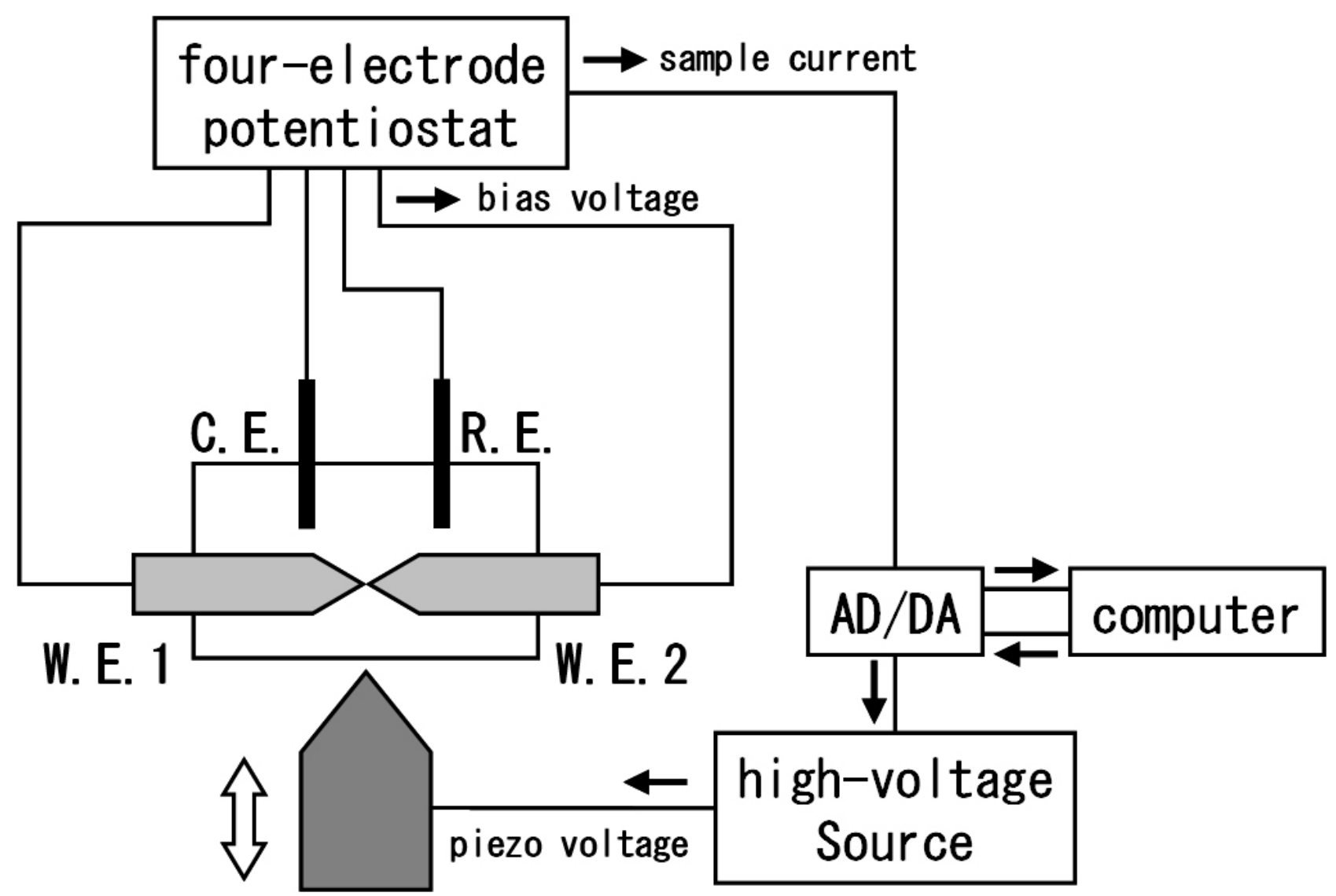


Figure4

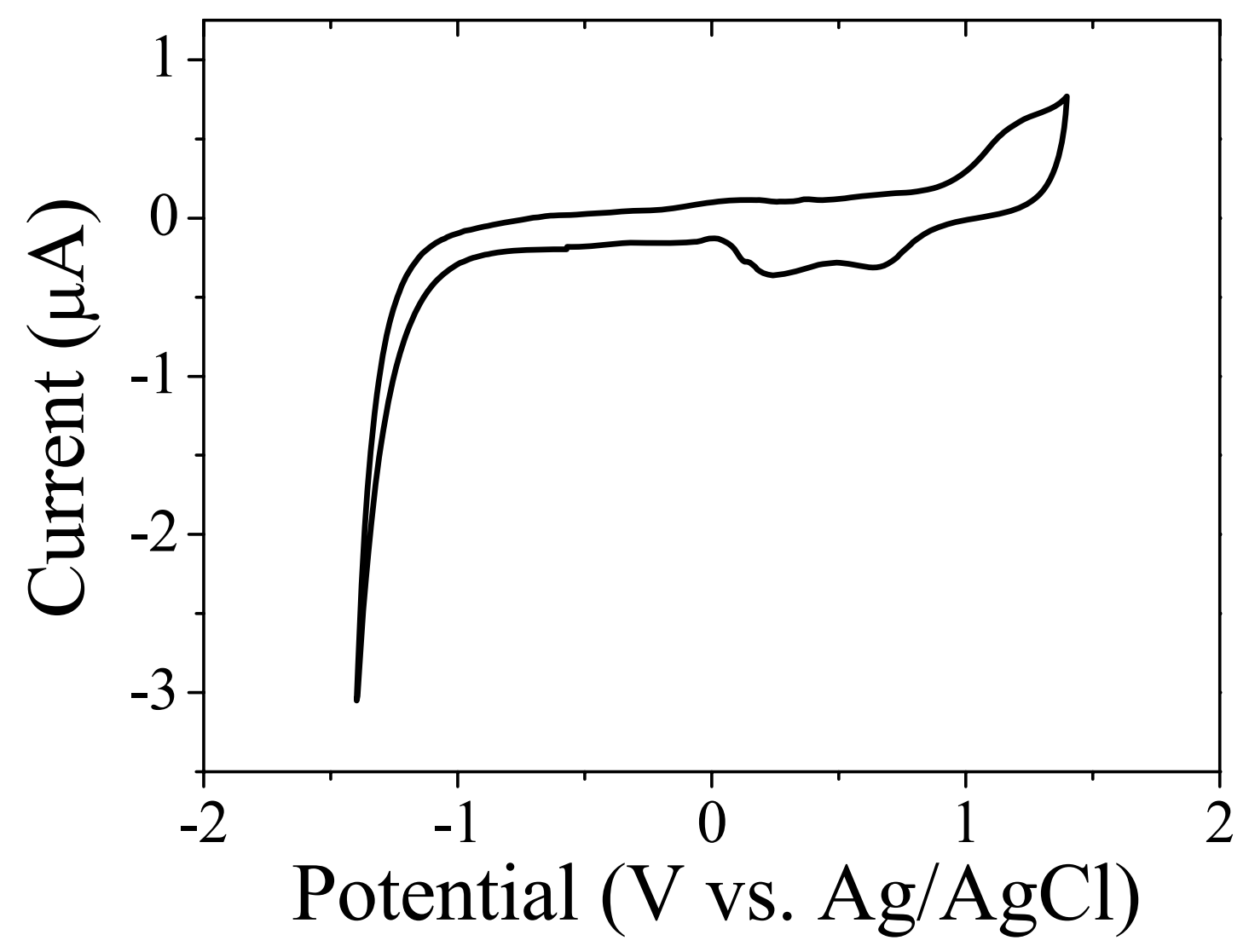


Figure5

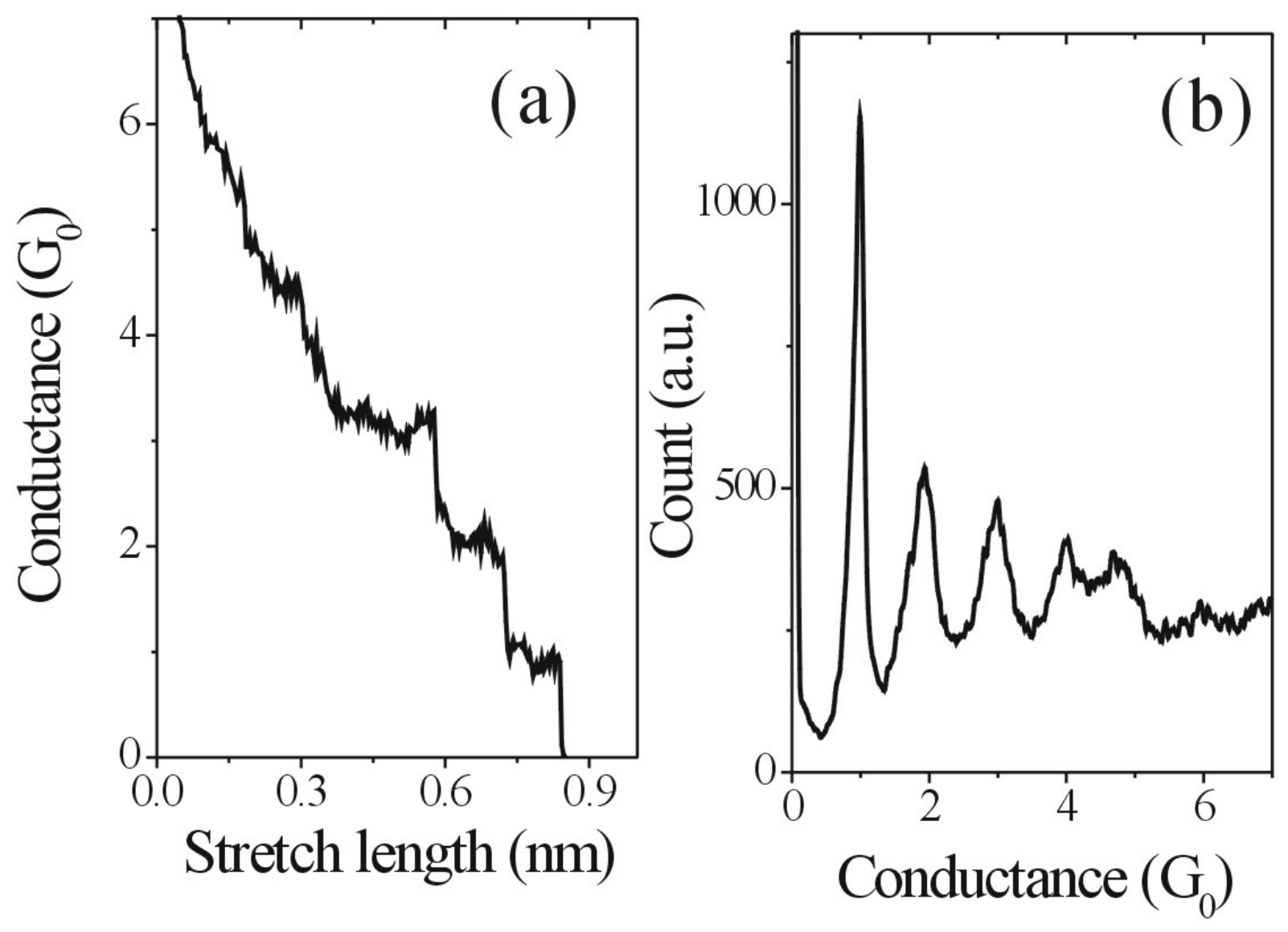


Figure6

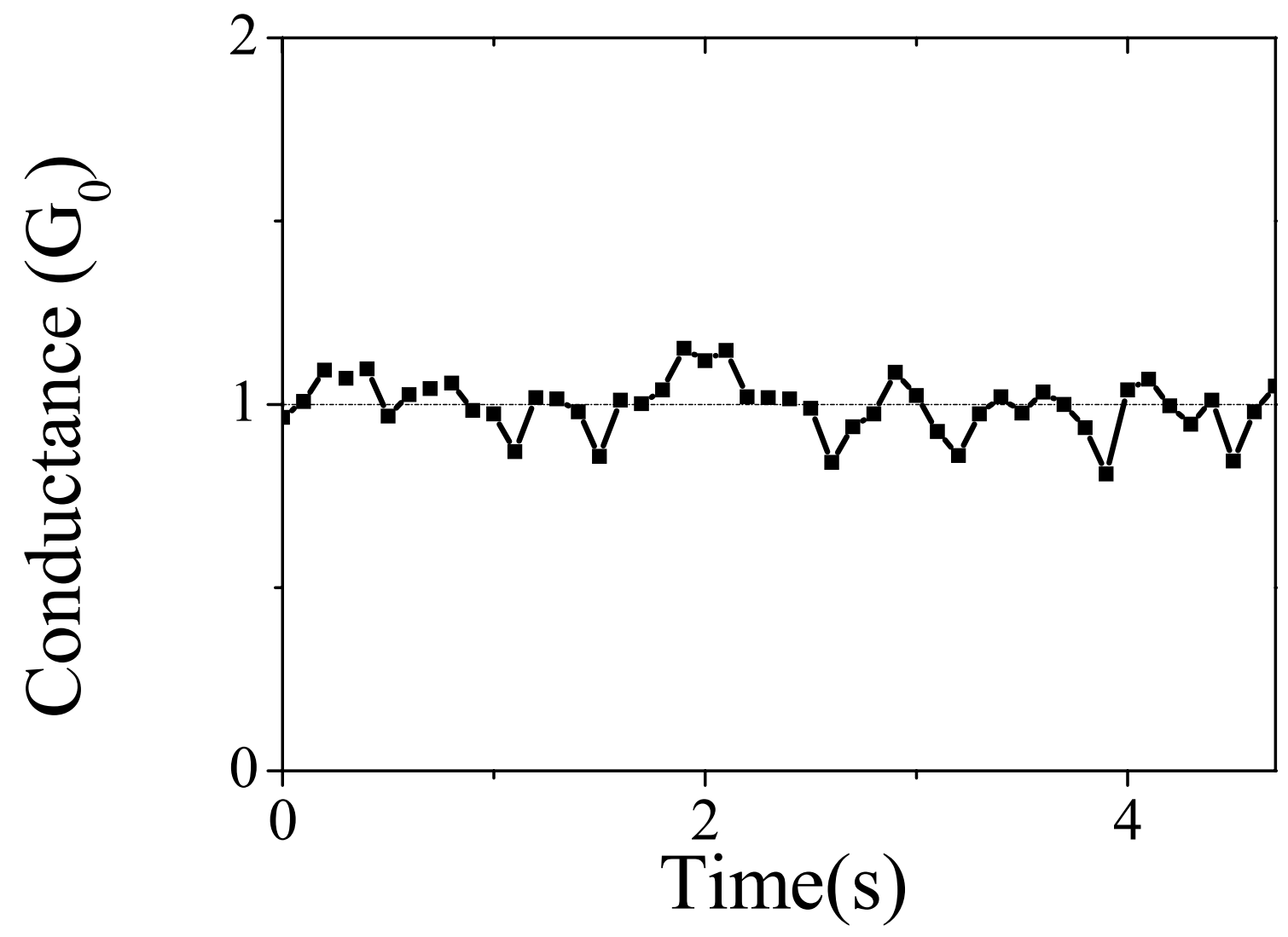

